\title{
Quantitative assessment of radiodermatitis through a non-invasive objective procedure in patients with breast cancer
}

\author{
MAIKO KITAJIMA $^{1}$, KASUMI MIKAMI ${ }^{1}$, YUKA NOTO ${ }^{1}$, CHIEKO ITAKI $^{1}$, YASUYO FUKUSHI ${ }^{2}$, \\ YOSHIKO HIROTA $^{3}$, YASUSHI MARIYA ${ }^{2}$, MEGUMI TSUSHIMA ${ }^{1}$, KEIICHI KATTOU $^{3}$ and TOMOHIRO OSANAI ${ }^{1}$ \\ ${ }^{1}$ Department of Nursing Science, Hirosaki University Graduate School of Health Science, Hirosaki, \\ Aomori 036-8564; ${ }^{2}$ Department of Radiology, Mutsu General Hospital, Mutsu, Aomori 035-8601; \\ ${ }^{3}$ Hirosaki Central Hospital, Hirosaki, Aomori 036-8188, Japan
}

Received April 20, 2019; Accepted September 19, 2019

DOI: $10.3892 / \mathrm{mco} .2019 .1948$

\begin{abstract}
Assessment of skin condition is necessary for providing advice regarding skin self-care to patients with breast cancer who have undergone radiation therapy. Acute and chronic phases of radiodermatitis were analyzed in patients using objective assessment tools in the present study. A total of 18 women who received radiation therapy for breast cancer were enrolled in the present study and their skin surface temperature (SST), hydration level of the skin surface (HL), melanin intensity (MI) and erythema intensity (EI) were measured prior to radiation therapy and six months thereafter. Furthermore, skin condition was assessed using the Common Terminology Criteria for Adverse Events (CTCAE). EI and MI levels at the irradiated site peaked upon the completion of radiation therapy and declined to baseline at 6 months. In contrast, SST levels were elevated at the irradiated site during radiation therapy $(\mathrm{P}<0.05)$ and plateaued after its completion. The same parameters in non-irradiated control sites remained unchanged during the study period. HL reached the minimum in irradiated and non-irradiated sites upon completion of radiation therapy. Although HL returned to baseline in the non-irradiated site 6 months after radiation therapy, it remained low in the irradiated site. No relationship between the CTCAE and EI level was observed. In conclusion, the present study demonstrated that objective assessment tools, including SST and EI levels, were useful for assessing skin condition during radiodermatitis. The combination of the CTCAE and objective assessment tools will enable a more accurate assessment of radiodermatitis.
\end{abstract}

Correspondence to: Dr Maiko Kitajima, Department of Nursing Science, Hirosaki University Graduate School of Health Science, 66-1 Hon-cho, Hirosaki, Aomori 036-8564, Japan

E-mail: kitajima@hirosaki-u.ac.jp

Key words: breast cancer, radiodarmatitis, skin surface temperature, erythema intensity, hydration level

\section{Introduction}

Radiation therapy for breast cancer has been established as a standard treatment after breast-conserving surgery (1). In postmastectomy radiation therapy, the dose on the skin surface ranges from 50 to $60 \%$ of the prescribed one, which is usually 2 Gy daily at the isocenter or the International Commission of Radiation Units reference point (2). Darby et al reported that survival rates improved and relapse rates for breast cancer decreased after radiation therapy (3). However, radiation therapy may have adverse effects on tissues or organs of patients receiving the same. Therefore, it is of great importance that medical staff supports skin self-care and adequately manages the symptoms of patients undergoing radiation therapy.

Radiodermatitis, an acute adverse effect, has been considered as one of the most frequent symptoms. Despite the skin-sparing benefits of contemporary techniques, most patients experience some degree of integumentary system reaction $(4,5)$. Symptoms usually appear a few weeks after the onset of radiation therapy, wherein the skin becomes red, dry, warm, and sore (6). To take care for such patients, medical staff needs to assess the skin condition in the irradiated area and educate the patients regarding skin lesion care according to the degree of skin reaction.

Generally, medical staff in Japan has used the Common Terminology Criteria for Adverse Events (CTCAE version 4.0) for skin evaluation (7). However, CTCAE is limited in its capability to assess detailed changes in skin condition because of its subjective nature. For example, under the 'Dermatitis radiation' classification, CTCAE defines 'Faint erythema or dry desquamation' and 'Moderate to brisk erythema' as Grade 1 and Grade 2, respectively. However, nurses frequently find it difficult to distinguish 'Faint' skin reactions from 'Moderate' one. Furthermore, the assessment of radiodermatitis using CTCAE might vary depending on the person assessing the skin reaction.

Our previous research identified skin surface temperature (SST) and erythema intensity (EI) as objective assessment tools that could clarify the skin condition in detail both during and after radiation therapy (8). However, our prior study included only a small sample size, and the measurements of those parameters were performed at only two points: Before and after radiation therapy. The present study therefore investigated the changes in skin condition among patients with 
breast cancer who underwent radiation therapy using objective assessment tools in greater detail.

\section{Patients and methods}

Subjects. A total of 18 patients with breast cancer undergoing radiation therapy after surgery were enrolled. This study was approved by the Committee for Medical Ethics of Hirosaki University (Hirosaki) and was conducted after obtaining written informed consent from each patient.

Radiation therapy was performed at Hirosaki Central Hospital using a linear accelerator (ClinaciX, Varian Medical Systems) employing 6-MV X-rays. Tangential irradiation technique with two non-parallel portals was used. The total target dose was 50 Gy in 25 fractions with a conventional schedule.

Measurements. The patients' skin condition was measured using a Multi-skin instrument ${ }^{\circledR}$ consisting of Mexameter ${ }^{\circledR}$ MX18, Corneometer ${ }^{\circledR}$ CM825, and Skin-Thermometer ${ }^{\circledR}$ ST500 (Courage + Khazaka Corporation) $(9,10)$ before, during, and six months after radiation therapy. These devices enable non-invasive measurement through pen-type probes and can evaluate four skin parameters. The measured parameters include SST, hydration level of the skin surface (stratum corneum) (HL), melanin intensity (MI), and EI. The measurement of HL was 10-20 $\mu \mathrm{m}$ of the stratum corneum to avoid the influence of deeper skin layers (e.g., from the blood vessels). Measurements using a $15 \mathrm{~mm}$ thick foil showed only $20 \%$ of the original measurement value (9). MI and EI were measured based on absorption/reflection of light. The Mexameter ${ }^{\circledR}$ MX18 probe is able to emit three specific light wavelengths $(568,660$ and $870 \mathrm{~nm}$ ), and the receiver measures the light reflected by the skin. The highly sensitive measurement gives broad-scale values (0-999) for melanin and erythema. Therefore, even the smallest changes in color become traceable (10). The quantity of light absorbed by the skin can be calculated. The MI among healthy Japanese women is reported to be approximately 100-200 (11,12). The measurement procedure was performed in a medical examination room that was maintained at a temperature of $25.4 \pm 0.6^{\circ} \mathrm{C}$ via an air conditioner.

Subjective evaluation index of acute radiodermatitis. The severity or grade of acute radiodermatitis was clinically evaluated according to the CTCAE version 4.0, Japanese Clinical Oncology Group version (7). CTCAE was developed by National Cancer Institute and is widely accepted throughout the oncology research community as the standard grading scale for adverse events. The radiation dermatitis index of the CTCAE consists of five scales (Table I). In this study, one radiologist who is a specialist of radiotherapy judged what CTCAE level of the subject's dermatitis and one nurse confirmed that throughout the period of this study.

Statistical analysis. All data are expressed as means \pm standard deviations and were analyzed using the SPSS version 22.0 software. Results were compared using repeated measures ANOVA followed by Bonferroni. Comparisons between the irradiated and contralateral side at the same time-point were performed using a two-sided paired t-test. $\mathrm{P}<0.05$ was considered to indicate a statistically significant difference.

\section{Results}

Patient characteristics. A total of 18 patients with breast cancer who underwent breast-conserving surgery and postmastectomy radiation therapy were enrolled. The patient characteristics are shown in Table II. The average age was $56.8 \pm 11.7$ years, and their breast cancer stages ranged from IA through IIIB. Moreover, the laterality of the primary lesion of six patients was right side, while that of the other 12 patients was left side. In the pathological classification, one patient was adenocarcinoma, not other specified, and 11 patients were ductal carcinoma. Women who had breast-conserving surgery for unifocal invasive ductal adenocarcinoma (excluding invasive carcinoma of classical lobular type) of any grade (1-3) were recruited. Nine patients received hormone administration, four received chemotherapy, and four received both hormone administration and chemotherapy.

Changes in parameters during radiation therapy. EI levels at the irradiated site gradually elevated during radiation therapy and peaked upon completion thereof, whereas those at the non-irradiated contralateral site remained unchanged (Fig. 1). EI levels at the irradiated site returned to baseline six months after cessation of the radiation therapy and were comparable to those at the non-irradiated site.

Similar to EI, MI levels at the irradiated site were elevated during radiation therapy but those at the contralateral non-irradiated site remained unchanged (Fig. 2). Upon completion of radiation therapy, MI levels at the irradiated sites were higher than those at the contralateral site $(\mathrm{P}<0.05)$. MI levels at the irradiated site subsequently declined to baseline six months after radiation therapy.

Fig. 3 shows the changes in HL at the irradiated and nonirradiated sites during and after radiation therapy. In contrast to EI and MI, HL at both sites was at its lowest upon completion of radiation therapy. HL at the non-irradiated control site recovered to baseline six months after radiation therapy, whereas that at the irradiated site remained lower than that at the control site.

As shown in Fig. 4, no difference in SST levels was observed between the irradiated and nonirradiated sites before radiation therapy. However, SST levels at the irradiated site became elevated during radiation therapy $(\mathrm{P}<0.05)$ and plateaued even after its cessation, whereas that at the non-irradiated site remained unchanged throughout the study. Even six months after radiation therapy, the SST levels were higher at the irradiated site than at the non-irradiated site $(\mathrm{P}<0.05)$.

Comparison between the CTCAE and EI levels. The CTCAE grades ranged from 0 to 2 in this study. EI levels ranged from 177 to 333 and from 119 to 283 when the CTCAE was Grade 1 and Grade 2, respectively.

\section{Discussion}

Our research revealed the degree of radiodermatitis for breast cancer patients using novel instrument however this is a small study. The major findings of the present study showed that EI and MI levels at the irradiated site peaked upon completion of radiation therapy and subsequently declined to baseline after six months. In contrast, SST levels at the irradiated site increased during radiation therapy and 
Table I. Dermatitis radiation index of CTCAE.

\begin{tabular}{lllll}
\hline Index & Grade 1 & \multicolumn{1}{c}{ Grade 2 } & Grade 3 & Grade 4 \\
\hline $\begin{array}{l}\text { Dermatitis } \\
\text { radiation }\end{array}$ & $\begin{array}{l}\text { Faint erythema or } \\
\text { dry desquamation }\end{array}$ & $\begin{array}{l}\text { Moderate to } \\
\text { brisk erythema; } \\
\text { patchy moist } \\
\text { desquamation, } \\
\text { mostly confined } \\
\end{array}$ & $\begin{array}{l}\text { Moist desquamation } \\
\text { in areas other than } \\
\text { skin folds and creases; } \\
\text { bleeding induced by } \\
\text { minor trauma or abrasion }\end{array}$ & $\begin{array}{l}\text { Life-threatening } \\
\text { consequences; skin } \\
\text { necrosis or ulceration } \\
\text { of full thickness } \\
\text { dermis; spontaneous } \\
\text { bleeding from involved } \\
\text { creases; moderate } \\
\end{array}$ \\
& & & site; skin graft indicated
\end{tabular}

CTCAE, Common Terminology Criteria for Adverse Events.

Table II. Subject characteristics $(\mathrm{n}=18)$.

\begin{tabular}{lr}
\hline Characteristics & Value \\
\hline Age (years) & $56.8 \pm 11.7$ \\
Disease stage & 10 \\
Stage IA & 5 \\
Stage IB & 1 \\
Stage IA & 2 \\
Stage IB & \\
Laterality of primary lesions & 7 \\
Right & 11 \\
Left & 1 \\
Adenocarcinoma, NOS & 1 \\
Ductal & 1 \\
Mucinous adenocarcinoma & 15 \\
Tubular adenocarcinoma & \\
Scirrhous adenocarcinoma & \\
Combination therapy & 9 \\
Hormone therapy & 4 \\
Chemotherapy & 4 \\
Hormone therapy and chemotherapy & \\
\hline
\end{tabular}

NOS, not otherwise specified.

plateaued after its completion. The same parameters at the nonirradiated control site remained unchanged throughout the study period. Moreover, HL levels at both the irradiated and nonirradiated sites were at their lowest upon completion of radiation therapy. HL at the nonirradiated sites returned to the baseline six months after radiation therapy, whereas that at the irradiated sites remained low. No relationship was observed between the CTCAE grade and EI level.

Duration of skin observation and self-care. Radiodermatitis has been associated with the integumentary system's response to planned ionizing radiation exposure, which causes depletion of stem cells from the basal layer of the epidermis (13). More intense erythema and hyperpigmentation have been shown to occur after 2-3 weeks of treatment $(14,15)$. The present study

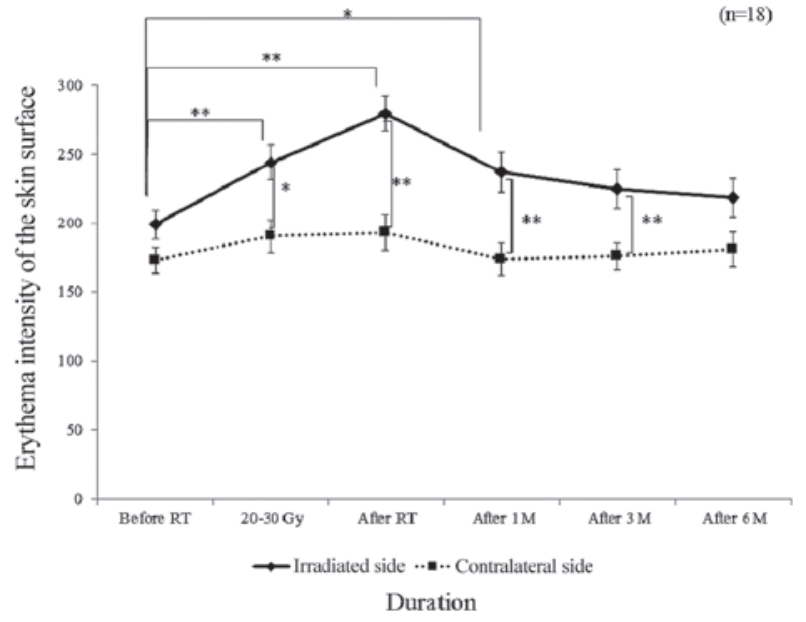

Figure 1. Erythema index in patients with breast cancer undergoing radiation therapy $(\mathrm{n}=18)$. ${ }^{*} \mathrm{P}<0.05,{ }^{* *} \mathrm{P}<0.01$. RT, radiation therapy; M, months.

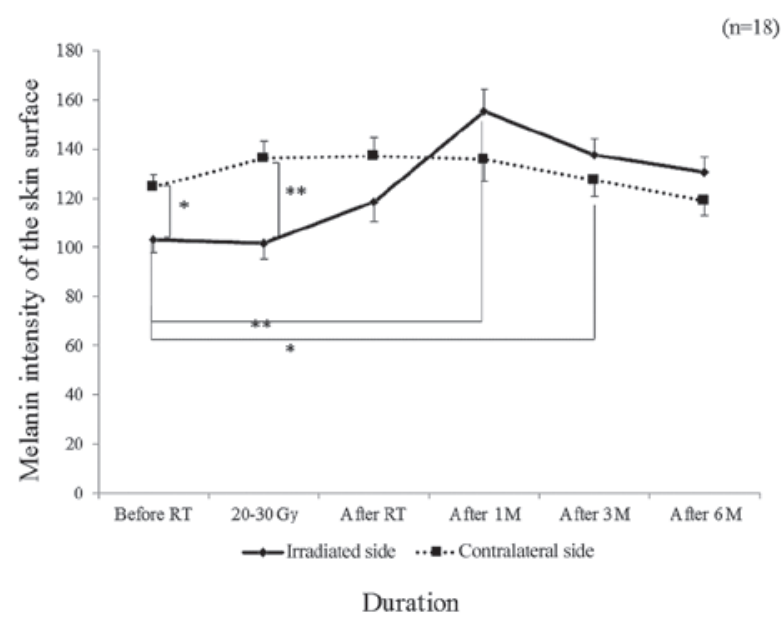

Figure 2. Melanin levels of patients with breast cancer undergoing radiation therapy $(\mathrm{n}=18) .{ }^{*} \mathrm{P}<0.05,{ }^{* *} \mathrm{P}<0.01$. $\mathrm{RT}$, radiation therapy; $\mathrm{M}$, months.

investigated changes in skin condition before and six months after the radiation therapy in patients with breast cancer. According to the objective assessment tools, SST, MI, and HL remained impaired six months after cessation of radiation therapy, although EI levels had already recovered after radiation therapy. 


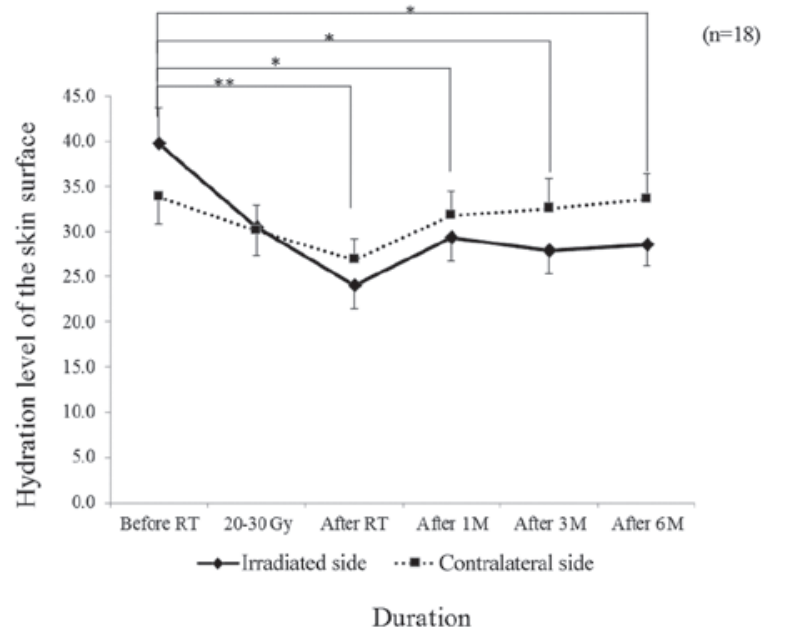

Figure 3. Hydration level of skin surface in patients with breast cancer undergoing radiation therapy $(\mathrm{n}=18) .{ }^{*} \mathrm{P}<0.05,{ }^{* *} \mathrm{P}<0.01$. RT, radiation therapy; M, months.

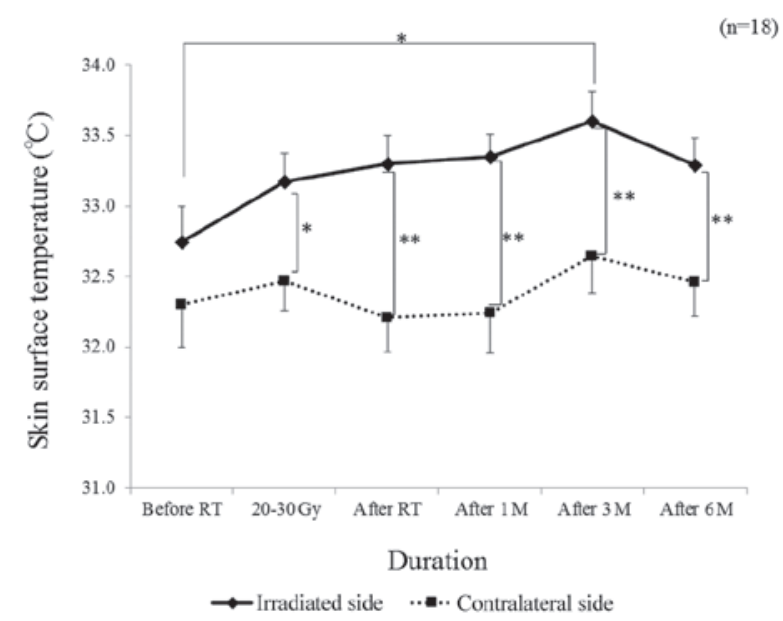

Figure 4. Skin surface temperature in patients with breast cancer undergoing radiation therapy $(\mathrm{n}=18) .{ }^{*} \mathrm{P}<0.05,{ }^{* *} \mathrm{P}<0.01$. $\mathrm{RT}$, radiation therapy; $\mathrm{M}$, months.

In addition, EI evaluated as CTCAE-Grade 1 overlapped with that evaluated as CTCAE-Grade 2. These findings suggest that even when skin erythema could no longer be observed visually, observation of skin condition should be continued for at least six months after radiation therapy. Functional changes, such as those to the sweating ability of the irradiated skin, might persist longer than readily visible morphological changes.

HL at the irradiated sites was significantly lower than that at the nonirradiated ones during and after radiation therapy. This might be related to impaired sweating due to sweat gland damage after irradiation. It seems reasonable to presume that basal skin layer damage is involved in prolonged recovery. This prolonged phenomenon strongly necessitates medical staff to provide patient education regarding skin hydration and usage of moisturizers. Moisturizers may facilitate skin fluid volume recovery, which can be determined by analyzing the difference between the presence and absence of moisturizer usage and the frequency of application. In a study evaluating radiodermatitis caused by accelerated partial breast irradiation (APBI) using high-dose-rate interstitial brachytherapy, changes in moisture were less severe and recovery was more rapid after APBI than after external beam radiation therapy (16). Accordingly, a difference in the appearance of dermatitis has been observed based on radiotherapy treatment. In this study, the subjects did not use moisturizer until after radiodermatitis occurred. Sekiguchi et al reported that the application of a heparinoid moisturizer for 2 weeks resulted in lower skin dryness and desquamation compared to the non-application thereof (17). Therefore, medical staff should advice their patients regarding the appropriate timing of moisturizer usage.

Inflammation and preventive strategy. SST levels at the irradiated sites were significantly higher than those at the nonirradiated sites six months after radiation therapy. Although this could have been caused by the inflammatory response after surgery, it is difficult to confirm the pathogenesis of the higher SST levels. We emphasize that even when EI levels at the irradiated site had normalized, inflammatory changes that needed self-care still persisted.

Incidence rates of dermatitis may be dependent on various factors. For example, reports have shown that patients with breast volume $>1,600 \mathrm{~cm}^{3}$ had more acute skin lesions (18), while incidence rates of radiodermatitis were correlated with the planning target volume. To anticipate the risk of adverse effects, nurses need to communicate with radiologists and radiological technologists regarding the treatment plan before radiation therapy. After radiation therapy initiation, instructions regarding the appropriate underclothing and method of skin cleaning provided by the nurses might prevent skin problems. Given that the patients' self-care habits could affect their skin condition, statements regarding patient self-care should henceforth be analyzed using objective assessment tools. Whelan et al reported that adverse effects like radiodermatitis and breast pain induced a decline in patient quality of life (QOL) (19). Thus, using some objective parameters to help patients understand their skin condition is imperative. In this study, we used noninvasive instruments to objectively assess the patients' skin condition objectively. Conversely, Fuzissaki et al used the Radiation Therapy Oncology Group and the World Health Organization scale together, whereas Partl et al employed the application of a new image-analysis tool $(20,21)$. These methods can help to assess a patient's skin without inter-observer variability. The assessment tool in this study has a great benefit for patients to monitor their skin changes without pain. In addition, patients who experienced stress at the beginning of radiotherapy had the same or increased levels of stress during and shortly after treatment (22). The assessment of objective parameters might help patients to maintain or improve their QOL and also provide them the opportunity to recognize their skin condition. Significant predictors of distress and emotional upset prior to radiation therapy included feelings of pessimism, cancer stage, and history of mastectomy (23). The level of distress among patients with breast cancer did not significantly vary until six months post-treatment, but it could span up to 12 months or more after the initial diagnosis (24). Therefore, we should also consider the patient's emotional state when providing an explanation regarding their skin condition and methods of self-care.

In conclusion, skin assessment with the instrument used in this study is useful to assess the details of the skin and explain 
patients regarding the need of good skin self-care. Moreover, we believe that the combination of CTCAE and objective assessment tools will enable a more accurate assessment of radiodermatitis.

\section{Acknowledgements}

We would like to thank all of the patients and medical staff who participated in this study. The authors would additionally like to thank Enago (www.enago.jp) for the English language review.

\section{Funding}

The present study was supported by JSPS KAKENHI (grant no. 26670951).

\section{Availability of data and materials}

Datasets used and/or analyzed during the current study are available from the corresponding author on reasonable request.

\section{Authors' contributions}

MK initiated the research. MK, KM, YN, CI, YF, YH, MT, and KK collected the data from the subjects. MK, KM, YN, CI, YF, YH, YM, MT, KK and TO analyzed the data. YM and TO drafted, reviewed and revised the manuscript. All authors read and approved the final manuscript.

\section{Ethics approval and consent to participate}

This study was approved by the Committee for Medical Ethics of Hirosaki University (Hirosaki) (approval no. 2013-65). All patients provided informed consent prior to study participation and for publication of any associated data.

\section{Patient consent for publication}

Not applicable.

\section{Competing interests}

The authors declare that they have no competing interest.

\section{References}

1. Gordils-Perez J, Rawlins-Duell R and Kelvin JF: Advances in radiation treatment of patients with breast cancer. Clin J Onco Nurs 7: 629-636, 2003.

2. Landberg T, Chavaudra J, Dobbs J, Gerard PJ, Hanks G, Horiot JC, Johansson KA, Möller T, Purdy J and Suntharalingam N: ICRU report 62 (Supplement to ICRU report 50). International Commission on Radiation Units and Measurements (ICRU, Bethesda, USA, 1999.

3. Early Breast Cancer Trialists' Collaborative Group (EBCTCG); Darby S, McGale P, Correa C, Taylor C, Arriagada R, Clarke M, Cutter D, Davies C, Ewertz M, et al: Effect of radiation therapy after breast-consercing surgery on 10-year recurrence and 15-year breast cancer death: Meta-analysis of individual patient data for 10,801 women in 17 randomised trials. Lancet 378 $1707-1716,2011$

4. De Conno F, Ventafridda V and Saita L: Skin problems in advanced and terminal cancer patients. J Pain Symptom Manage 6: 247-256, 1991.
5. Porock D and Kristjanson L: Skin reactions during radiation therapy for breast cancer: The use and impact of topical agents and dressings. Eur J Cancer Care (Engl) 8: 143-153, 1999.

6. Dodd MJ: Managing the side effects of chemotherapy and radiation therapy. 2nd edition. UCSF Nursing Press, San Francisco, California, , USA, 2001.

7. US Department of Health and Human Services: Common Terminology Criteria for Adverse Events (CTCAE). V4.03, 2010. https://evs.nci.nih.gov/ftp1/CTCAE/CTCAE_4.03/ CTCAE_4.03_2010-06-14_QuickReference_5x7.pdf.

8. Fukushi Y, Kitajima M, Itaki C, Noto Y, Mikami K, Hirota Y, Katto K and Mariya Y: Changes in skin surface temperature and erythema intensity during and after radiation therapy for breast cancer patients. Radiat Emerg Med 3: 47-51, 2014.

9. Heinrich U, Koop U, Leneveu-Duchemin MC, Osterrieder K, Bielfeldt S, Chkarnat C, Degwert J, Häntschel D, Jaspers S, Nissen HP, et al: Multicentre comparison of skin hydration in terms of physical-, physiological- and product-dependent parameters by the capacitance method (Corneometer CM 825). Int J Cosmet Sci 25: 45-53, 2003.

10. Park ES, Na JI, Kim SO, Huh CH, Youn SW and Park KC: Application of a pigment measuring device-Mexameter-for the differential diagnosis of vitiligo and nevus depigmentosus. Skin Res Technol 2: 298-302, 2006.

11. Tamai Y, Tsuji M, Wada K, Nakamura K, Hayashi M, Takeda N, Yasuda K and Nagata C: Association of cigarette smoking with skin colour in Japanese women, Tob Control 23: 253-256, 2014.

12. Nagata C, Konish K, Tamura T, Wada K, Hayashi M, Takeda N and Yasuda K: Skin pigmentation is inversely associated with insulin resistance in healthy Japanese women. Diabetes Metab 42: 368-371, 2016.

13. Eaton L and Tipton $\mathrm{J}$ (eds): Putting evidence into practice: Improving oncology patient outcomes. 1 st edition. Oncol Nurs Society, Pittsburgh, PA, USA, 2009.

14. Blackmar A: Radiation-induced skin alteration. Medsurg Nurs 6: 172-175, 1997.

15. Knopf MT and Sun Y: A longitudinal study of symptoms and self-care activities in women treated with primary radiation therapy for breast cancer. Cancer Nurs 28: 210-218, 2005.

16. Tanaka E, Yamazaki H, Yoshida K, Takenaka T, Masuda N, Kotsuma T, Yoshioka Y and Inoue T: Objective and longitudinal assessment of dermatitis after postoperative accelerated partial breast irradiation using high-dose-rate interstitial brachytherapy in patients with breast cancer treated with breast-conserving therapy: Reduction of moisture deterioration by APBI. Int J Radiat Oncol Biol Phys 81: 1098-1104, 2011.

17. Sekiguchi K, Ogita M, Akahane K, Haga C, Ito R, Arai S, Ishida Y, Tsukada Y and Kawamori J: Randomized, prospective assessment of moisturizer efficacy for the treatment of radiation dermatitis following radiotherapy after breast-conserving surgery. Jpn J Clin Oncol 45: 1146-1153, 2015.

18. Vicini FA, Beitsch PD, Quiet CA, Keleher A, Garcia D, Snider HC, Gittleman MA, Zannis VJ, Kuerer H, Whitacre EB, et al: First analysis of patient demographics, technical reproducibility, cosmesis, and early toxicity: Results of the American society of breast surgeouns mammosite breast brachytherapy trial. Cancer 104: 1138-1148, 2005.

19. Whelan JT, Levine M, Julian J, Kirkbride P and Skingley P: The effects of radiation therapy on quality of life of women with breast carcinoma: Results of a randomized trial. Ontario Clinical Oncology Group. Cancer 88: 2260-2266, 2000.

20. Partl R, Lehner J, Winkler P and Kapp KS: Testing the feasibility of augmented digital skin imaging to objectively compare the efficacy of topical treatments for radiodermatitis. PLoS One 14: e0218018, 2019

21. Fuzissaki MA, Paiva CE, Gozzo TO, Maia MA, Canto PPL and Maia YCP: Is there agreement between evaluators that used two scoring systems to measure acute radiation dermatitis? Medicine (Baltimore) 98: e14917, 2019

22. Sehlen S, Hollenhorst H, Schymura B, Herschbach P, Aydemir U, Firsching $M$ and Dühmke E: Psychosocial stress in cancer patients during and after radiotherapy. Strahlenther Onkol 179: $175-180,2003$.

23. Soh SJ, Schnur JB, Sucala M, David D, Winkel G and Montgomery GH: Distress and emotional well-being in breast cancer patients prior to radiotherapy: An expectancy-based model. Psychol Health 27: 347-361, 2012.

24. Lester J, Crosthwaite K, Stout R, Jones NR, Holloman C, Shapiro C and Andersen LB: Women with breast cancer: Self-reported distress in early survivorship. Oncol Nurs Forum 42: E17-E23, 2015. 